\title{
Enhancer RNAs: A Class of Long Noncoding RNAs Synthesized at Enhancers
}

\author{
Tae-Kyung Kim¹, Martin Hemberg ${ }^{2}$, and Jesse M. Gray ${ }^{3}$ \\ ${ }^{1}$ The University of Texas Southwestern Medical Center, Department of Neuroscience, Dallas, Texas 75390-9111; ${ }^{2}$ Boston \\ Children's Hospital, Department of Ophthalmology, Boston, Massachusetts 02215; ${ }^{3}$ Genetics Department, Harvard Medical \\ School, Boston, Massachusetts 02115 \\ Correspondence: taekyung.kim@utsouthwestern.edu
}

Recent studies have revealed that active enhancers are transcribed, producing a class of noncoding RNAs called enhancer RNAs (eRNAs). eRNAs are distinct from long noncoding RNAs (IncRNAs), but these two species of noncoding RNAs may share a similar role in the activation of mRNA transcription. Emerging studies, showing that eRNAs function in controlling mRNA transcription, challenge the idea that enhancers are merely sites of transcription factor assembly. Instead, communication between promoters and enhancers can be bidirectional with promoters required to activate enhancer transcription. Reciprocally, eRNAs may then facilitate enhancer-promoter interaction or activate promoter-driven transcription.

The functional contribution of enhancers to gene expression has been well shown over the past three decades. The mechanisms by which enhancers influence gene expression, however, remained poorly understood. Recent technological advances have made it possible to observe, on a genome-wide scale, the molecules and mechanisms that govern enhancer function. We know that enhancers recruit general coactivators, such as p300/CBP, and they show a common chromatin signature. This signature includes high levels of monomethylation at histone $\mathrm{H} 3$ lysine 4 (H3K4me1), but low levels of the promoter-specific H3K4me3 mark (Fig. 1). Using CBP and histone methylation patterns to identify neuronal enhancers, our study revealed that several thousand enhancers can recruit RNA polymerase II (Pol II) and transcribe noncoding RNAs upon neuronal activation (Kim et al. 2010). The transcripts, which we termed enhancer RNAs (eRNAs), have since been independently confirmed in many different cell types and species, suggesting that eRNA synthesis is not unique to neurons, but more likely a universal cellular mechanism involved in governing enhancer function.

Enhancer RNAs are clearly distinguishable from the canonical long noncoding RNAs (lncRNAs) whose functions have been better characterized. A first distinction is that although lncRNAs were broadly defined based on the presence of $\mathrm{H} 3 \mathrm{~K} 4 \mathrm{me} 3$ at their promoters, eRNAs can be produced from enhancers without detectable levels of H3K4me3. This difference may stem from the 10- to 100fold lower expression levels of eRNAs relative to lncRNAs, as $\mathrm{H} 3 \mathrm{~K} 4 \mathrm{me} 3$ levels generally correlate with gene expression level. Second, unlike the promoters of lncRNAs and protein-coding genes, enhancers show little bias in the direction of transcription initiation. Third, whereas lncRNAs undergo maturation processes such as splicing and polyadenylation, eRNAs are shorter $(<2 \mathrm{~kb})$, with little evidence of being consistently spliced or polyadenylated. The lack of polyadenylation was inferred by the fact that eRNAs were first detected from analysis of total cellular RNA (using total RNA-seq) in neurons, but not observed in polyadenylated RNA (using mRNA-seq). Polyadenylated eRNAs, however, have been reported or implied from the analysis of other nonneuronal cell types. Despite some of these differences between stereotypical eRNAs and lncRNAs, we and others have observed a relatively small number of genomic loci that cannot be easily classified either as enhancers or promoters of IncRNAs because of the presence of both $\mathrm{H} 3 \mathrm{~K} 4 \mathrm{me} 3$ and H3K4mel marks. These loci could represent a distinct class of enhancers or

Editors: C. David Allis, Marie-Laure Caparros, Thomas Jenuwein, and Danny Reinberg

Additional Perspectives on Epigenetics available at www.cshperspectives.org

Copyright (C 2015 Cold Spring Harbor Laboratory Press; all rights reserved; doi: 10.1101/cshperspect.a018622

Cite as Cold Spring Harb Perspect Biol 2015;7:a018622 
T.-K. Kim et al.
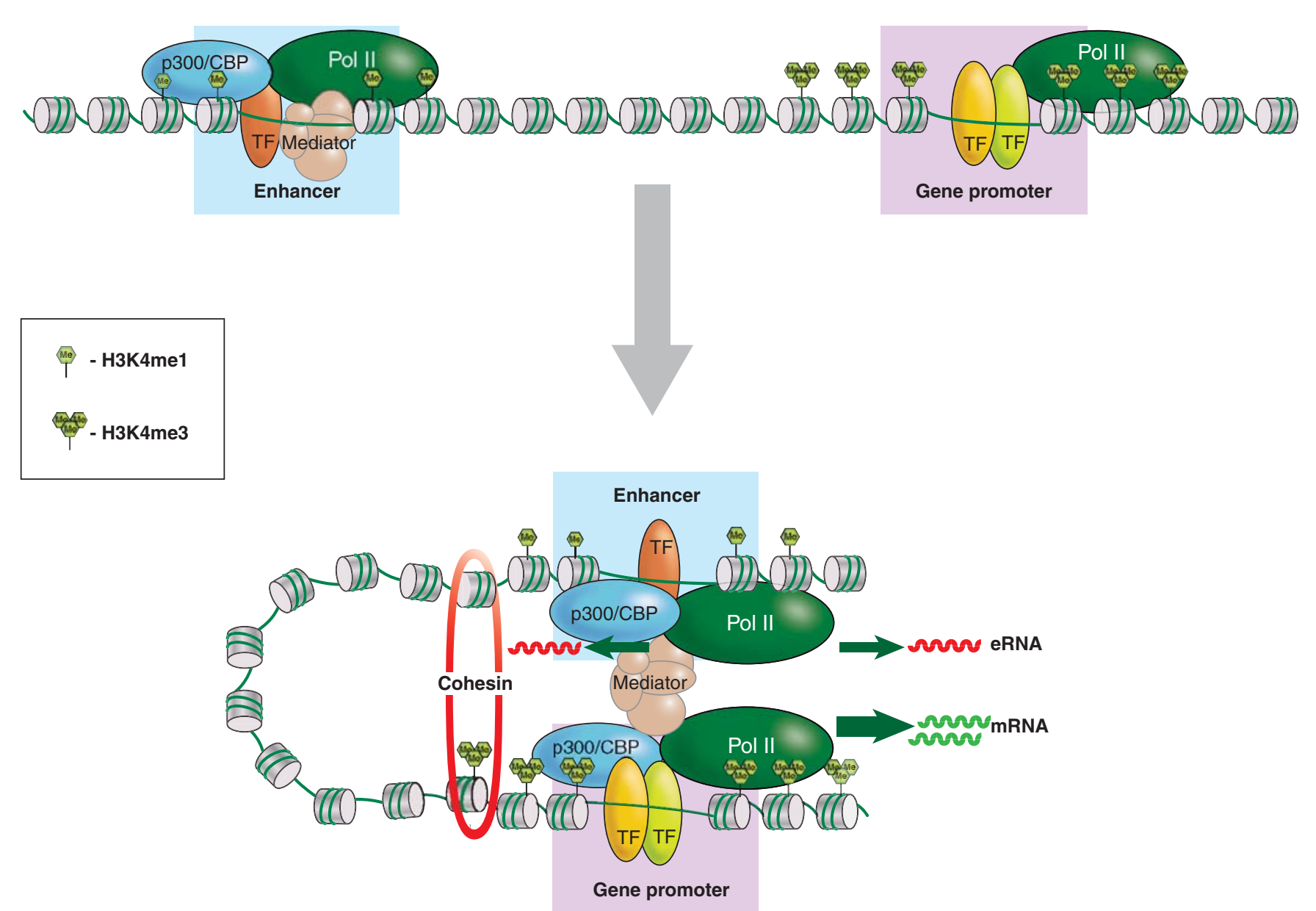

Figure 1. eRNA synthesis and function. During transcriptional activation, coactivator (e.g., p300/CBP) and RNA Pol II bind to a subset of enhancers and bidirectionally transcribe eRNAs. Chromatin looping between the enhancer and promoter will bring eRNAs near the target gene promoter to allow coordinate activation. Some eRNAs (e.g., eRNAs expressed from ER- $\alpha$-bound enhancers in human breast cancer cells) facilitate and/or stabilize specific enhancer-promoter looping, in part by interacting with cohesin.

may suggest that enhancer and promoter designations are only useful to a point. The distinction between enhancer and promoter may simply be a quantitative one concerning the expression levels of transcripts. Indeed, protein-coding promoters have been reported to act as enhancers, regulating other nearby promoters.

The major question raised by the discovery of eRNAs relates to whether transcriptional activity at enhancers contributes to enhancer function. Several lines of circumstantial evidence suggest that eRNA synthesis is a regulated process and not mere transcriptional noise. Upon neuronal stimulation, only a subset of enhancers produces eRNAs, and this subset tends to be located near mRNAs that are strongly induced (Kim et al. 2010). Based on this observation, we propose that eRNA-producing enhancers are actively engaged in promoting the expression of target genes in response to stimulus-induced signaling. This hypothesis has been supported by correlative studies of eRNAs in various cell types. Moreover, kinetic analysis of eRNAs in lipopolysaccharide-activated macrophages showed that eRNA synthesis precedes transcription of adjacent protein-coding genes, suggesting an active role for eRNAs in the regulation of the target gene. Additional support comes from a recent siRNA-mediated knockdown study of human lncRNAs, inferring that they contribute to the activation of surrounding protein-coding mRNAs (Lai et al. 2013). Although it has not been clearly shown whether the lncRNAs with enhancer-like function are derived from enhancer regions, these GENCODE IncRNAs differ from eRNAs in that they are typically processed by splicing and polyadenylation, as well as having high levels of $\mathrm{H} 3 \mathrm{~K} 4 \mathrm{me} 3$ at their promoters. Nonetheless, the combination of these two independent findings-eRNA derived from functionally defined enhancers and lncRNAs showing enhanc- 
er-like function-implies that non-protein-coding regions throughout the genome may produce transcripts with specific regulatory functions and have a more active role than previously anticipated in gene expression.

Supporting this notion, recent studies have provided more direct evidence demonstrating that at least some eRNAs are functionally important for target gene expression. The knockdown of several eRNAs caused decreased expression of nearby target genes (Lam et al. 2013; Li et al. 2013; Melo et al. 2013). In addition, the artificial tethering of eRNAs upstream of a minimal promoter in a plasmidbased reporter system enhances the reporter gene expression. These results are consistent with the proposed role of eRNAs in transcriptional activation. Intriguingly, the activating function of eRNAs appears to be sequence- or strand-specific, although the critical determinants for this specificity have not been identified (Lam et al. 2013). In other experiments in human breast cancer cells, eRNAs expressed from estrogen receptor $\alpha$ (ER- $\alpha$ )-bound enhancers increase the strength of specific enhancer-promoter looping, in part by interacting with cohesin ( $\mathrm{Li}$ et al. 2013). The lncRNAs with enhancer-like function mentioned above were also shown to mediate chromatin looping, but through the interaction with a mediator complex (Lai et al. 2013).

Although these results collectively suggest that chromatin looping is an important regulatory step by which these distinct classes of activating lncRNAs commonly act (Fig. 1), there might be other functions of eRNAs. Our study, focusing on the neuronal $\operatorname{Arc}$ gene and enhancer, showed that eRNA synthesis requires the presence of an intact Arc gene promoter (Kim et al. 2010); that is, detectable levels of eRNAs are not synthesized when the Arc gene promoter is deleted, although RNA Pol II and transcription factors still bind to the enhancer. One possibility is that without its promoter, the Arc enhancer is missing an unknown factor required for its own transcription activity. Such an unknown factor would be present at the promoter and enable eRNA synthesis at the enhancer only when the enhancer is brought within close proximity with the promoter through a looping mechanism. These results challenge the standard model of unidirectional enhancer-promoter interactions in which enhancers act on promoters. Instead, enhancer and promoter activation may require feedback, with each contributing elements of the protein complement required for activation of the other. In this scenario, it is unlikely that the chromatin looping is facilitated entirely by eRNAs, as the eRNA synthesis would occur only after the enhancerpromoter looping. The chromatin looping would also keep nascent eRNAs in the proximity of target promoters, potentially providing an elegant way of preventing eRNAs from activating nonspecific target genes. A nascent eRNA transcript might then facilitate recruitment of activators to the promoter, acting as a scaffold for assembly of activating proteins. Because eRNAs are generally unstable, the specificity of eRNA function would come in part from its short half-life, preventing nonspecific activation away from its local site of synthesis, once synthesis was complete. It also needs to be pointed out that the act of eRNA transcription, in addition to the eRNA transcript itself, could have a specific biological function. For example, transcriptionally engaged RNA polymerase II could recruit chromatin modifiers to enhancers, stabilizing an enhancer domain in an active state.

The discovery and emerging functional roles of eRNAs certainly expand the growing regulatory capacity of noncoding RNAs. These findings not only illustrate a more complex role of cis-regulatory sequences than previously appreciated, but also provide an exciting avenue of future research in unraveling the intricate layers of gene regulation that are intertwined with lncRNAs, cis-regulatory sequences, epigenetic modifications, and three-dimensional chromatin configuration.

\section{ACKNOWLEDGMENTS}

We thank Drs. M.E. Greenberg and G. Kreiman for their guidance and support. This work was supported by the Whitehall Foundation (T-K.K.), and Welch Foundation (T-K.K.), and the Klingenstein Fund (T-K.K.).

\section{REFERENCES}

Kim TK, Hemberg M, Gray JM, Costa AM, Bear DM, Wu J, Harmin DA, Laptewicz M, Barbara-Haley K, Kuersten S, et al. 2010. Widespread transcription at neuronal activity-regulated enhancers. Nature 465: $182-187$.

Lai F, Orom UA, Cesaroni M, Beringer M, Taatjes DJ, Blobel GA, Shiekhattar R. 2013. Activating RNAs associate with Mediator to enhance chromatin architecture and transcription. Nature 494: 497-501.

Lam MT, Cho H, Lesch HP, Gosselin D, Heinz S, Tanaka-Oishi Y, Benner C, Kaikkonen MU, Kim AS, Kosaka M, et al. 2013. Rev-Erbs repress macrophage gene expression by inhibiting enhancer-directed transcription. Nature 498: 511-515.

Li W, Notani D, Ma Q, Tanasa B, Nunez E, Chen AY, Merkurjev D, Zhang J, Ohgi K, Song X, et al. 2013. Functional roles of enhancer RNAs for oestrogen-dependent transcriptional activation. Nature 498: 516520.

Melo CA, Drost J, Wijchers PJ, van de Werken H, de Wit E, Oude Vrielink JA, Elkon R, Melo SA, Leveille N, Kalluri R, et al. 2013. eRNAs are required for 53-dependent enhancer activity and gene transcription. Mol Cell 49: 524-535. 


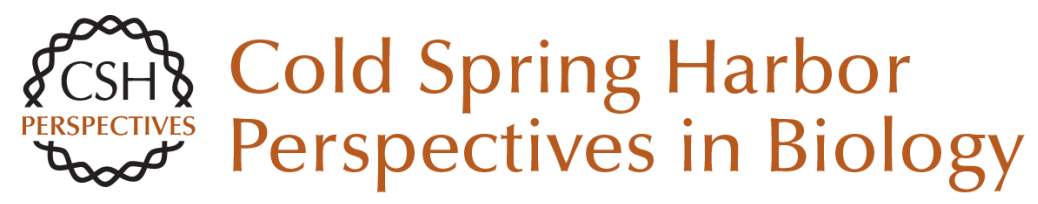

\section{Enhancer RNAs: A Class of Long Noncoding RNAs Synthesized at Enhancers}

Tae-Kyung Kim, Martin Hemberg and Jesse M. Gray

Cold Spring Harb Perspect Biol 2015; doi: 10.1101/cshperspect.a018622

\section{Subject Collection Epigenetics}

Metabolic Signaling to Chromatin

Shelley L. Berger and Paolo Sassone-Corsi

Histone and DNA Modifications as Regulators of

Neuronal Development and Function Stavros Lomvardas and Tom Maniatis

Histone Modifications and Cancer James E. Audia and Robert M. Campbell

Epigenetics and Human Disease Huda Y. Zoghbi and Arthur L. Beaudet

Induced Pluripotency and Epigenetic Reprogramming

Konrad Hochedlinger and Rudolf Jaenisch

\section{Long-Range Chromatin Interactions} Job Dekker and Tom Misteli

RNAi and Heterochromatin Assembly Robert Martienssen and Danesh Moazed

Dosage Compensation in Drosophila John C. Lucchesi and Mitzi I. Kuroda
Epigenetic Determinants of Cancer

Stephen B. Baylin and Peter A. Jones

Maintenance of Epigenetic Information Geneviève Almouzni and Howard Cedar

A Structural Perspective on Readout of Epigenetic Histone and DNA Methylation Marks Dinshaw J. Patel

The Necessity of Chromatin: A View in

Perspective Vincenzo Pirrotta

Germline and Pluripotent Stem Cells Wolf Reik and M. Azim Surani

Comprehensive Catalog of Currently Documented Histone Modifications Yingming Zhao and Benjamin A. Garcia

Epigenetic Regulation of Chromatin States in Schizosaccharomyces pombe Robin C. Allshire and Karl Ekwall

Histone Variants and Epigenetics Steven Henikoff and M. Mitchell Smith

For additional articles in this collection, see http://cshperspectives.cshlp.org/cgi/collection/

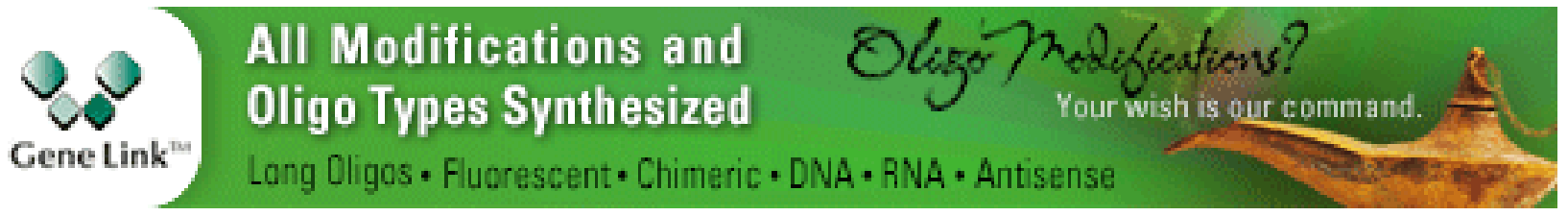

Copyright (C) 2015 Cold Spring Harbor Laboratory Press; all rights reserved 
For additional articles in this collection, see http://cshperspectives.cshlp.org/cgi/collection/

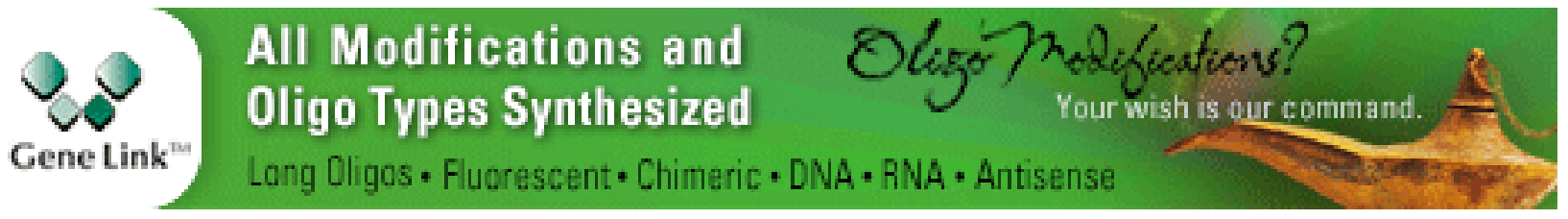

Copyright @ 2015 Cold Spring Harbor Laboratory Press; all rights reserved 